\title{
Guest Editorial: Parallelism in Algorithms and Architectures
}

\author{
Michael A. Bender • Seth Gilbert
}

Published online: 21 September 2011

(C) Springer Science+Business Media, LLC 2011

This special issue originated from select papers from the 21st ACM Symposium on Parallelism in Algorithms and Architectures (SPAA), which was held August 11-13, 2009 in Calgary, Canada. The papers were selected by the editors based on evaluations from the program committee. The authors were invited to submit extended journal versions of their SPAA papers, and these submissions were rigorously evaluated using the standard review process for the journal. We thank the authors and reviewers for their hard work.

\section{M.A. Bender (凶)}

Department of Computer Science, Stony Brook University, Stony Brook, NY 11794-4400, USA e-mail: bender@cs.stonybrook.edu

M.A. Bender

Tokutek, Inc., Lexington, MA, USA

url: http://www.tokutek.com/

S. Gilbert

Department of Computer Science, National University of Singapore, COM2-03-23, 21 Lower Kent

Ridge Road, Singapore 119077, Singapore

e-mail: seth.gilbert@comp.nus.edu.sg 